九州大学学術情報リポジトリ

Kyushu University Institutional Repository

\title{
Clustering Adaptive Elephant Herd Optimization Based Data Dissemination Protocol for VANETs
}

Dwivedy, Bhoopendra

G. L. Bajaj Institute of Technology \& Management

\section{Anoop Kumar Bhola}

Department of Computer Science, Banasthali vidyapith

C.K. Jha

Department of Computer Science, Banasthali vidyapith

https://doi.org/10.5109/4742126

出版情報 : Evergreen. 8 (4)，pp.812-820，2021-12. 九州大学グリーンテクノロジー研究教育センター バージョン：

権利関係 : 


\title{
Clustering Adaptive Elephant Herd Optimization Based Data Dissemination Protocol for VANETs
}

\author{
Bhoopendra Dwivedy ${ }^{*}$, Anoop Kumar Bhola ${ }^{2}$, C.K. Jha ${ }^{3}$ \\ ${ }^{1}$ G. L. Bajaj Institute of Technology \& Management, Greater Noida, India \\ ${ }^{2}$ Department of Computer Science, Banasthali vidyapith, Rajasthan, India \\ ${ }^{3}$ Department of Computer Science, Banasthali vidyapith, Rajasthan, India \\ *Author to whom correspondence should be addressed: \\ E-mail: bdwivedy@gmail.com
}

(Received February 16, 2021; Revised September 24, 2021; accepted October 3, 2021).

\begin{abstract}
VANETs (Vehicular Ad hoc Networks) have pulled in enormous considerations because of their real-time application and business value. Due to the limited bandwidth of the wireless interface, dynamic topology, frequently disconnected networks, the communication between vehicles is a challenging task. Clustering is seen as one of the possible solutions to achieve effective communication in VANETs; this research proposes a Clustering Adaptive Elephant Herd Optimization (CAEHO) technique for VANETs. The proposed CAEHO protocol is used to form optimized clusters for robust communication. Cluster head $(\mathrm{CH})$ selection depends upon the fuzzy logic method by selecting parameters like connectivity levels, lane weighting, direction and speeds of vehicles. Based on a fitness function, these parameters are utilized to choose the optimal route between sender and receiver. The NS2 platform is used to implement the proposed work then it is contrasted with previous techniques such as Ant Colony Optimization algorithm (ACO) and Improved Whale Optimization algorithm (IWOA) respectively. Significantly, the CAEHO protocol enhances the packet delivery ratio, throughput and comparatively reduces overhead than other routing protocols.
\end{abstract}

Keywords: CAEHO protocol, EHO, Improved whale optimization algorithm, clustering, ACO and NS2 platform.

\section{Introduction}

Recently, the Intelligent Transportation Systems (ITSs) are used to transfer information between vehicles, which enhance the productivity and safety with minimal traffic congestion. The advanced applications of ITSs provide the services connected to traffic and transport management. The uses of ITSs enable safer and more coordinated route information. So vital mechanism of ITSs is Vehicular Ad hoc Networks (VANETs) ${ }^{12,24)}$.

In the research community, the area that has recently caught great attention is vehicular networks. Therefore the number of useful applications and algorithms are proposed, some algorithms are based on heuristics also ${ }^{20}, 23$. For media content sharing, trip planning and information retrieval with the means of transport crash evasion and other expensive requests are used in VANETs. A mobile sensor network in vehicles establishes the environmental conditions and other monitor roads. The vehicular networks are the "delivery networks" that transfer data from remote sensor nets to Internet servers ${ }^{2,10)}$. The communication among vehicles (sender to receiver) is the main challenge in VANETs. So here, multi-hop and wireless mode of data transmission are required. From many studies, it can be gathered that an efficient VANETs protocol solves these common issues for data transmission ${ }^{4,5)}$. The solution to some problems are stated, but some are not yet provided by different approaches so far ${ }^{15,22)}$.

A lot of studies related to the protocols for data communication verify the robust data packets delivering and applications that rely on a reliable vehicular methodology ${ }^{9,16)}$. The road users use robustly and rapidly the data in VANET communication, which is supported by various routing protocols ${ }^{19)}$.There are many problems in roadside communication like link breakdown on the routing paths, high-rise buildings or other obstacles in between routes and wireless links instability; in each case routing protocol plays a significant role ${ }^{8,21)}$. The data transfer in VANETs is discussed in many studies like Artificial Intelligence (AI) techniques, clustering algorithms, GW swarm optimization technique, Ant colony optimization algorithm and other heuristic 
approaches $^{6,7)}$.

\subsection{Problem Statement}

In VANETs data is propagated through the exchange of messages between the nodes. Hence, propagating data to the intended node incorporates many major issues in VANET such as Traffic delay/congestion, security and road condition etc. If vehicle driver is equipped with incomplete information (like vehicle speed, orientation of vehicle around them) then it results the driver may make wrong decisions (like lane changing, breaking or over speeding etc.).

The VANET may consist of high density vehicles, base stations (BS), intra cluster and inters cluster routing. In VANET, the node forwards traffic information to other node through intra routing manner and it forwards to BS using neighboring nodes through inter cluster routing manner. As the quantity of messages between nodes increases, it influences the performance of VANET. So the essential point of consideration for data delivery in VANET is routing algorithms to improve performance. So an Intelligent Transportation Systems (ITS) can be considered by deploying various applications between the vehicle to vehicle communications, which will improve the road safety and security.

\subsection{Aim and Advantages of the paper}

- To improve AODV protocol with the help of cluster head $(\mathrm{CH})$ selection mechanism; based on position, velocity and heuristic techniques.

- $\quad$ To find traffic density per unit time with the help of probabilistic distribution (poison distribution) with reference to an Observation point.

- To reduce the number of control overhead messages in VANET scenario.

- A heuristic technique like Elephant Herd Optimization algorithm is used to find the optimal path between cluster heads.

- By using Network Simulator (NS2) with the proposed CAEHO protocol, the performance is analyzed in term of throughput, overhead and data delivery ratio.

The proposed protocol of CAEHO via data dissemination of VANET is analyzed. Section 2 explains the newest investigation reviews. The detailed explanation of CAEHO model and results are illustrated in section $3 \& 4$, finally section 5 concludes the paper.

\section{Literature Review}

Many routing protocols have been proposed in previous literature. Any routing protocol means to scan the optimum route between vehicles. Following two types of approach is used for routing purpose.

- Proactive approach: - These are table-driven approach for example DSDV (Destination Sequenced Distance Vector), and OLSR (Optimized Link State Routing).

- Reactive approach: - These are on-demand routing protocols, here they establish path when required or when a source wants to send a message to the destination. Like Ad-hoc on-demand distance vector routing (AODV), Dynamic Source Routing (DSR) protocol, AOMDV protocol ${ }^{16)}$.

Following are the reasons for choosing an on-demand routing protocol in VANET.

a) Route discovery procedure as required.

b) It uses loop-free, single path/multipath approach ${ }^{20)}$.

So in VANET reactive protocols are used .The excellent data dissemination protocol for VANETs presented in the existing studies is analyzed in this section. The mechanized proposal of cluster heads and clustering of nodes is based on Evolutionary Game Theoretic (EGT) framework and was proposed by Ammara Anjum Khan et al. ${ }^{1)}$ that accomplishes the VANETs with cluster stability. The Lyapunov function has been used to test the stability and equilibrium point systematically.

The optimal dropbox deployment algorithm (ODDA) was advocated by Jianping $\mathrm{He}$ et al. $^{11)}$ where the hypothetical structure for accurate release time computation is used. In the VCPS (Vehicular Cyber-Physical Systems) environment, the efficient data dissemination between different devices for secure clustering was advocated by RasmeetS. Bali et al., ${ }^{18)}$. For trust computation, among other devices, the varying transmission characteristic of vehicles defines according to a different trust metric, and then the global and local levels are evaluated. For creating secure clusters, the critical parameter with a current security level of vehicles is established by the trust metric. This method designs fast clustering for trust establishment.

In vehicular FoG computing and congestion avoidance scenario, the emergency message dissemination schemes were presented by Ata Ullah et al. ${ }^{3)}$. The message congestion scenarios managed the FoG-assisted VANET architecture in a similar vein. The comparison of congestion avoidance highlights strengths and weaknesses. The multiple worths of Discrete Particle Swarm Optimization (DPSO) were carried out by Manisha Chahal et al. ${ }^{13)}$ for the identification of an optimal path in VANETs. Here the performance metrics like routing overhead, packet delivery ratio and average 
throughput is analyzed by extensive simulations. In partially connected VANETs, connectivity-aware data dissemination (CADD) approach was proposed by Zhiyuan $\mathrm{Li}$ et al. ${ }^{25)}$ to enhance the data transmission capacity. The node forwarding capability estimation introduces a different metric in the CADD protocol. The various matrices show the efficiency of the protocol for data dissemination. The existing solutions with CADD protocol results have delivered better outcome in case of protocol overhead, transmission delay and PDR.

Recently, many investigation works have talked about VANETs data dissemination. Because of its dynamic characteristics, network transmission is low. The challenging task is to discover an optimal path for data dissemination in VANETs. The information-centric VANETs obtained from Content-Centric Networks (CCN) where many studies propose to improve transmission performance. For assisting the data dissemination, the drop boxes are extremely useful in vehicular networks. It minimizes the data delivery delay that increases the contact probabilities between vehicles. For data dissemination, the efficient and stable routes provided by control protocols are clustering. The frequent cluster reformation is created by quick network topology variations in VANETs that provides route stability.

The beauty of proposed approach is Clustering and is seen as one of the possible solution to achieve effective communication in VANETs. So some heuristic optimization techniques like Particle Swarm Optimization and elephant herd optimization technique are used for clustering and cluster head $(\mathrm{CH})$ selection. Here, the $\mathrm{CH}$ node acts as a candidate (relay) node to forward data from source to destination. Cluster head (CH) selection depends on the fuzzy logic method by selecting parameters like connectivity levels, lane weighing, direction, and vehicles' speeds. These parameters are utilized to choose the optimal route between the sender and receiver based on a fitness function.

Moreover, the unicast protocols and broadcast protocols are the most popular existing approaches. In VANETs, the most popular protocol is a unicast protocol for data storing. In contrast, the broadcast scheme provides an efficient modulation and coding system. Next to that, more heuristic methods, including PSO, GSO and ACO algorithm could also be incorporated in the above methods. The routing algorithm of situation-Aware Multi-constrained QoS (SAMQ) is introduced for VANETs. The QoS parameters set are improved via an optimal path between two nodes. In VANET, the data dissemination is analyzed with the usage of CAEHO protocol. The deficiency error is minimized, and it measures the reliability according to the multiple criteria. The main contribution of the paper is described below.

\section{Proposed Methodology}

The proposed CAEHO protocol is the ideal strategy in fast VANET for effective data dissemination. This paper depends on the clustering process with CAEHO protocol for the data dissemination in VANETs. For better data dissemination, it is required to decrease the link breaks between vehicles. For this reliable path, we wish to put the steady clusters. And for steady clusters we use the population based optimization technique like elephant herd optimization method. After above enhancement when our method is compared with previous methods like IWOA (Improved Whale optimization algorithm) and ACO (Ant Colony Optimization) on network simulator-2, the time is minimized up to $60 \%$ even in different density and high-velocity environment.

\subsection{Clustering process}

The designated cluster head $(\mathrm{CH})$ links the members of each cluster at the intra-cluster level. The multi-hop link is used to connect the cluster heads at the inter-cluster level. Here multi-hop inter-cluster communication protocol is used between adjacent cluster heads to exchange their status. Finally, all cluster members' aggregate the information by cluster heads broadcast. Under the high data load of beacon transmissions, we use contention-free MAC. Contention-free MAC combines the synchronization of both inter-cluster and intra-cluster channel. The high bandwidth efficiency is provided by intra-cluster aggregation protocol. Adjacent cluster heads' communication is more reliable map dissemination in the aggregation stage which creates the channel as an efficient bandwidth. The clusters are formed in various ways represented in Fig.1.
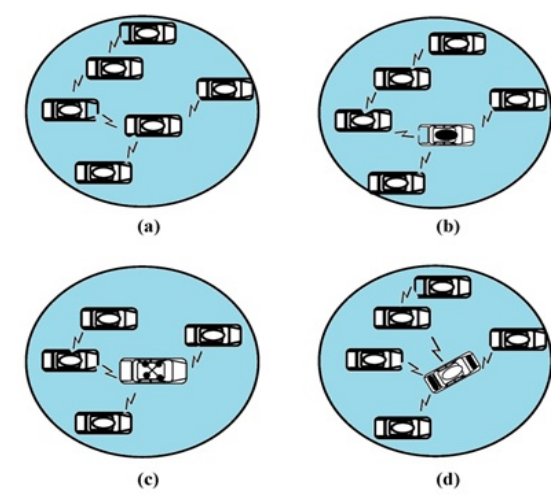

Fig.1: Various types of Clustering Process

\subsubsection{CH Selection Process}

We consider cluster formation in VANETs as an optimization problem. So the optimization technique is used to Form Clusters which is based on position, velocity and Whale parameters. After Clustering, Cluster 
head selection is an important aspect. Selection of Cluster head $(\mathrm{CH})$ based on delay, energy consumption and cooperation rate. The probability of each vehicle is calculated using the fitness value and the vehicle with the highest probability is selected as the cluster head.

Our objective is to reduce number of clusters so Clustering-based Adaptive Elephant Herd Optimization (CAEHO) technique is used for optimum clustering.

This $\mathrm{CH}$ will act as candidate node and will be able to forward the traffic information between nodes.

As the Cluster-head $(\mathrm{CH})$ is evaluated, it is broadcasted among nodes. For any node within that cluster radius to join, the $\mathrm{CH}$ broadcasts and invites automatically. The broadcast of $\mathrm{CH}$ message for all the nodes within the vicinity, invites node to join the cluster. Based on the continuous link to its cluster $\mathrm{CH}$, members can evaluate their connection, which is carried out periodically to confirm its status in terms of cluster affiliation. The re-clustering procedure may also be initiated, which take us back to the previous step if CMs fail to receive communication from $\mathrm{CH}$. Cluster stability depends on the criterion of a $\mathrm{CH}$ selection. The $\mathrm{CH}$ selection also depends upon the fuzzy logic method in which parameters like connectivity levels, lane weighting, direction and speeds are utilized ${ }^{14)}$, all are explained as follows.

\subsubsection{Lane Weight}

In an urban environment, the vehicular traffic splits at each intersection into three as;

Left Turn (LT) - Takes the left turn on the left-most lane

Right Turn (RT) - Takes the right turn on the right-most lane.

No Turn (NT) - The middle one never takes a turn in traffic in this lane but goes straight. The vehicle's lane of travel is determined by lane weighting aids ${ }^{17)}$. The general lane weighing (LW), (TNL) total no. lanes and (NLTF) no. of lanes in traffic direction is explained as follows:

$$
L W_{k}=\frac{1}{T N L} \times N L T F_{k}
$$

Here the hypothesis is cluster head is in the center of route. While outer lanes, the half the total weights allocated to the middle lane Eq. 1 and Eq.2.

$$
\begin{array}{rlrl}
L W_{k}= & \frac{1}{T N I} \times N L T F_{k} & & \text { for } T N L<2 \\
& \frac{2}{T N I} \times N L T F_{k} & \text {, for } T N I>2 \\
& \frac{2}{T N I} \times N L T F_{k} & \text {, for } T N I=2
\end{array}
$$

Consider the four lane scenario as shown in following figure (Fig.2)

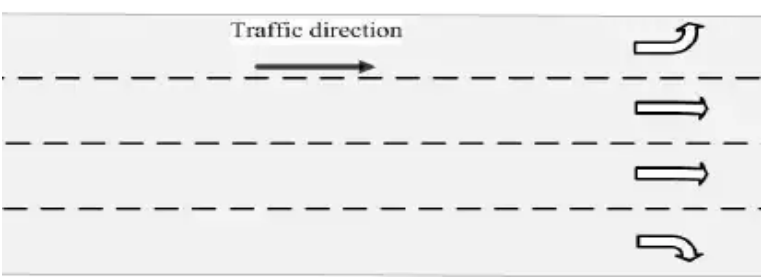

Fig.2: TNL, Left Turn, Right Turn and No Turn

According to scenario $\mathrm{TNL}=4, \mathrm{LT}=1, \mathrm{RT}=1$ and $\mathrm{NT}=2$

So $\mathrm{LW}_{\mathrm{LT}}=0.25$

$\mathrm{LW}_{\mathrm{RT}}=0.25$ and $\mathrm{LW}_{\mathrm{NT}}=0.50$ by equation number -2

Where TNL is total no. of Lanes, LT implies Left turn,

RT implies Right turn and LW, $\mathrm{LW}_{\mathrm{LT}}, \mathrm{LW}_{\mathrm{RT}}, \mathrm{LW}_{\mathrm{NT}}$ are Lane weighing, Lane weighing Left turn, Lane weighing Right turn , Lane weighing No turn respectively.

Based on every traffic flow (TF), the overall Network Connectivity Level (NCL) can be calculated. Based on the overall NCL, i refer to the number of connected nodes.

$\alpha_{i}(t)=\sum_{j} A(i, j, t)$

For the TF of the vehicle, the potential connected neighbouring vehicle is 'j' and connectivity level (CL) $\beta$.

$$
\begin{aligned}
& \beta_{i}(t)=\sum_{j} A\left(i, j_{T F}, t\right) T F \\
& N C L_{i}(t)=\beta_{i}(t)+\left(\alpha_{i}(t) \times L W T F\right)
\end{aligned}
$$

The below equation explains the level of normalized network connectivity

$$
N C L_{\text {inorm }}=\frac{N C L_{i}}{\max \left(N C L_{x}\right)}
$$

The distance $\delta_{\mathrm{i}}$ between vehicles is evaluated as:

$\delta_{i}=\frac{\sum_{j} \sqrt{\left(x_{j}-x_{i}\right)^{2}+\left(y_{j}-y_{i}\right)^{2}}}{N V}$

The other vehicles in the same TF denoted as $\chi_{\mathrm{i}}$ and the average absolute distance, between vehicles $i$.

$X_{i}=\frac{\sum_{j T F} \sqrt{\left(x_{j}-x_{i}\right)^{2}+\left(y_{j}-y_{i}\right)^{2}}}{N V_{T F}}$

The below equation explains the ADL for the ith vehicle in TF.

$$
\begin{aligned}
& A D L_{i}=X_{i}+\left(\delta_{i} \times L W_{T F}\right) \\
& A D_{\text {inorm }}=\frac{A D L_{i}}{\max \left(A D L_{x}\right)}
\end{aligned}
$$

Based on $\mathrm{CH}$ candidate is to calculate the average difference velocities of all vehicles. The overall Average Velocity Level (AVL), $\sigma_{\mathrm{i}}$.

$\sigma_{i}=\frac{\sum_{N V} \mid \text { vel }_{i}-\text { vel }_{j} \mid}{N V}$ 


$$
\rho_{i}=\frac{\sum_{N V T F}\left|v e I_{i T F}-v e I_{j T F}\right|}{N V_{T F}}
$$

$$
A V L_{i}=\rho_{i}+\left(\sigma_{i} \times L W_{T F}\right)
$$

The AVL is calculated for the ith vehicle in TF.

$$
A V L_{\text {inorm }}=\frac{A V L_{i}}{\max \left(A V L_{x}\right)}
$$

Based on their neighbour cluster-head candidacy Level (CHL) weights between other parameters such as position, velocity and so on are broadcasted and computed the equation (15) explains the highest weighted CHL. The vehicle is chosen by $\mathrm{CH}$

$$
C H L_{i}=w_{1} * N C L_{\text {inorm }}(t)+w_{2} * A D L_{\text {inorm }}+w_{3} * A V L_{\text {inorm }}
$$

The full description of the proposed algorithm is described in the following part.

\subsubsection{Adaptive Elephant herd optimization algorithm}

The recently established swarm intelligence algorithm is Elephant herding optimization algorithm, which is based on the performance of elephant herding. The clans are the categorization of Elephant population by each position of the elephant's denotes on a solution. Under the leadership of a matriarch is the natural living behaviour of elephants in one clan (Called as optimal fitness). Several male elephants in each generation identify the worst fitness function values with the solutions. In two years ago, the EHO algorithm was presented, and it is applied in many optimization issues. The three-tank system with level control to tune most favourable proportional-integral-derivative (PID) controller in the EHO algorithm. In-home energy management system, the alone aerial vehicle path planning is used pro neighbourhood discovery in multifaceted social networks. Next to a population of $\mathrm{N}$ solutions initialization then the answer is categorized into $\mathrm{n}$ clusters (clans). If one is done randomly, then the clusters are about a similar dimension. Based on the cluster's most excellent solution, every solution $\mathrm{j}$ of the cluster $\mathrm{i}$ alter and it is shown in the below equation.

$x_{\text {new }}, c_{i}, j=x_{c i}, j+\alpha^{*}\left(x_{\text {best }}, c_{i}-x_{c i}, j\right) * r$

Hence, a new solution $\mathrm{j}$ in a cluster is $x_{n e w} c_{i, j}$ and

denotes the existing solution. The best clan solution is represented as $X_{b e s t}, C_{i}$. The parameter represents the influence of the matriarch is $c_{i}, \alpha \in[0,1]$. The population diversity is enhanced using the random interval is $r \in[0,1]$. The $l, X_{\text {best }}, C_{i}, \quad$ is the best solution in cluster ${ }^{11)}$.

$x_{\text {new }}, c_{i}=\beta * x_{\text {center }}, c_{i}$,

Therefore, the parameter of the algorithm is $\beta \in[0,1]$ and manage the pressure of the $X_{\text {center }}, C_{i}$ $x_{\text {center }}, c_{i}, d=\frac{1}{n_{c i}} \times \sum_{i=1}^{n_{c i}} x_{c i}, l, d$

Hence $\mathrm{d}^{\text {th }}$ dimension (problem definition is $\mathrm{D}$ ) is $1 \leq d \leq D$ and the clan i with the number of elephants are $n_{c i}$.

In each cluster, by generating random solutions with the exploration was implemented and substituting the worst answers. The below equation explains the new solutions as:

$x_{\text {worst }}, c_{i}=x_{\min }+\left(x_{\max }-x_{\min }+1\right) *$ rand ,

From the equations inferior and superior boundaries of the search space are $X_{\min }$ and $X_{\max }$. The random digit chosen from uniform allocation is rand $\in[0,1]$.

The algorithm of EHO joint with k-means is introduced in this paper. The EHO algorithm searches the optimal positions of the centroids. The number of attributes is equivalent to the problem dimension; the number of clusters $\mathrm{k}$ is described by instances multiplied. The equation (17) explains the fitness function. There before each fitness functions evaluation by improving the K-means algorithm. In one iteration of the k-means algorithm with each generated solution is adjusted. The EHO algorithm generates the centroids cluster in the instances. The equation (16) shows the solution update by computing the instances. For data clustering, the suggested AEHO method pseudo-code with k-means is summarized in following, and the flow chart is illustrated in Fig.3:

So flow chart of elephant herd optimization is illustrated in the following Figure. Here we initialize the population of EHO with dimension six and parameters $\alpha$, $\beta$ are taken as .5, 0.1 respectively. The three-tank system with level control to tune most favourable proportional-integral-derivative (PID) controller in EHO algorithms like PID-1 and PID-2 is initialized by equation (16). Then by evaluating equation (13), (15) \& (17) will generate new position for best fit. 


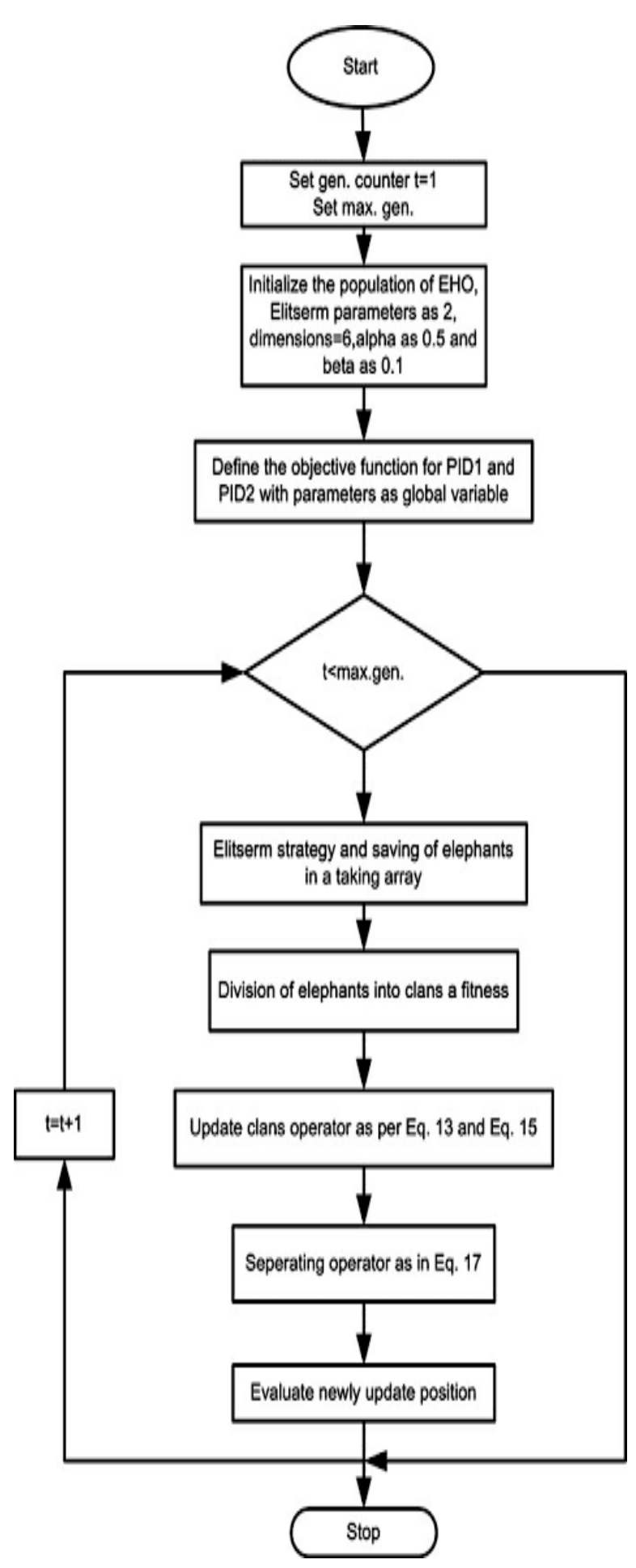

Fig.3: Flowchart of EHO algorithm

\subsubsection{Pseudo-code for best path selection:}

\section{Start}

Initialization of counters $=1$ to MaxGen

\section{Population initialization}

The clusters by dividing data instances

Use k-means algorithm to update the population

\section{Repeat}

According to their fitness to sort all the elephants

For

The population in all clusters $C_{i}$ do

For

The cluster $C_{i}$ with all solutions $j$

do

Update $X_{c i, j}$ and generate $x_{\text {new }}, c_{i, j}$ by Eq. (18)

If $x_{c i, j}=x_{\text {best }}, c_{i}$

Then Update $X_{c i, j}$ and

generate $X_{\text {new }}, C_{i, j}$ by Eq. (19)

The clusters with centroids from

$X_{c i, j}$ divide data instances

Use the k-means algorithm to update solution $X_{C i, j}$

\section{End if}

\section{End for}

\section{End for}

For

In the population with all clans $C_{i}$

do

By using Eq. (17) to replace the worst elephant in clan $C_{i}$

The clusters are divided by data instances

By the k-means algorithm to update generated solution

\section{End for}

By the newly updated positions to evaluate population

Until $\mathbf{t}<$ MaxGen

Return the optimal solution

\section{Results and discussions}

The performance metrics such as average cluster lifetime, $\mathrm{CH}$ duration, cluster size and other parameters are achieved by VANET structure of the proposed system and was simulated on the platform of NS2. Every exposure $1000 \mathrm{~m}$ radius, the two RSUs with $1500 \mathrm{~m}$ length and $24 \mathrm{~m}$ width (each direction in12 m) by two-way lane. Every imitation life form replicated $1000 \mathrm{~s}$ real-time, the permitted utmost vehicle speed of $50 \mathrm{~km} / \mathrm{h}$ $(13.89 \mathrm{~m} / \mathrm{s})$, and presumptuous the chance nature of vehicular speeds are utilized. To know their positions on the road segment by all the vehicles in the system are up to with global positioning system (GPS) and OBU. Approximately $250 \mathrm{~m}$ radius used by the communication 
range of a car, thereby using QPSK modulation frequency $5.9 \mathrm{GHz}$.

One of the vital vehicle synchronizations is RSU with the grouping of vehicles dependent on its movement direction. If information exchange is not possible from ping and $\mathrm{CH}$, then communication From RSUs is received. After that, lower distance with the vehicle aligns itself with an RSU. The fuzzy logics initiated after that stage of $\mathrm{CH}$ assortment. The $\mathrm{CH}$ nomination with the crucial component is NCL, ADL and AVL.

Figure 4 shows two scenarios such as cluster head selection index with input parameters and second average no. of nodes concerning cluster head. So $\mathrm{CH}$ selection with the impact of various input parameters is shown in this Figure. In $\mathrm{CH}$, the numbers of directly connected vehicles have the highest NCL, which is a crucial parameter in selection. The second most important parameter is distance level between CHs. Third is the average velocity between the vehicles, which shows the AVL parameter. For better connectivity, the NCL index should be high with the implication of less ADL and AVL, as shown in the following Figure.

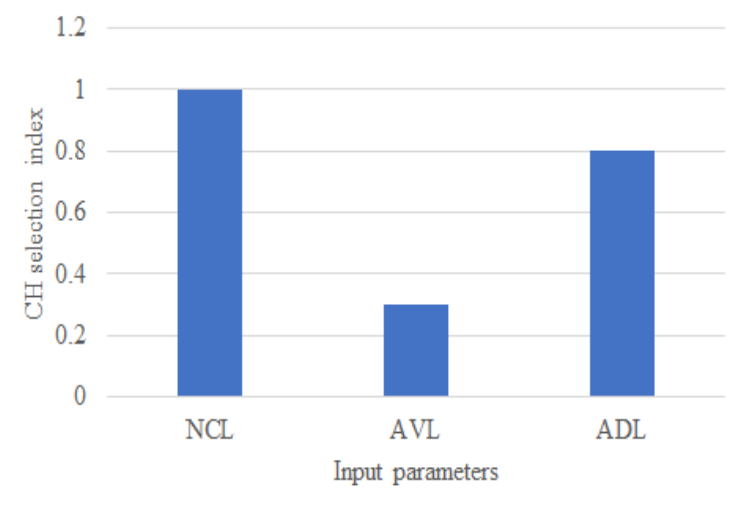

(a)

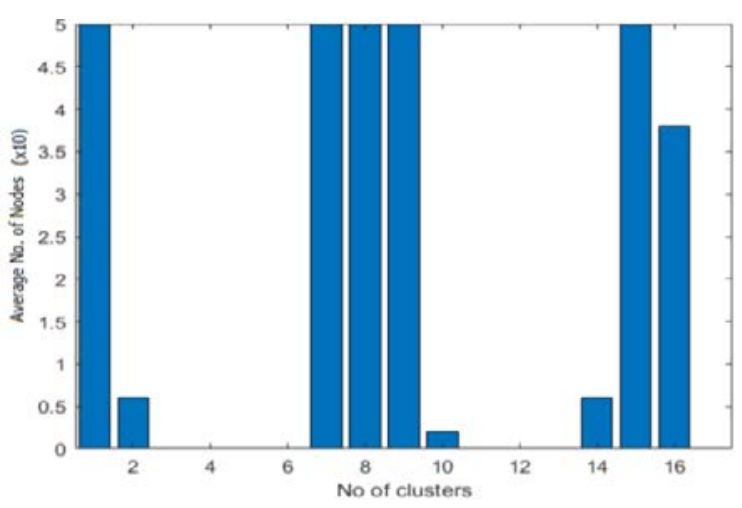

(b)

Fig.4: Analysis of (a) CH selection index with input parameters and (b) Node counts in a cluster

In the VANET network, node counts in each cluster are indicated in Figure 5-6. Figure 5 shows overhead, throughput and delivery ratio concerning the average number of nodes. In figure 5(a) as the number of nodes increases the overhead increases simultaneously, but our method shows less overhead as compared to other methods. In figure 5(b) as the number of nodes increases the throughput varies and is also suitable as compared. In figure 5(c) as the number of nodes increases the delivery ratio increases as compared to Ant Colony Optimization algorithm (ACO) and Improved Whale Optimization algorithm (IWOA) ${ }^{20)}$.

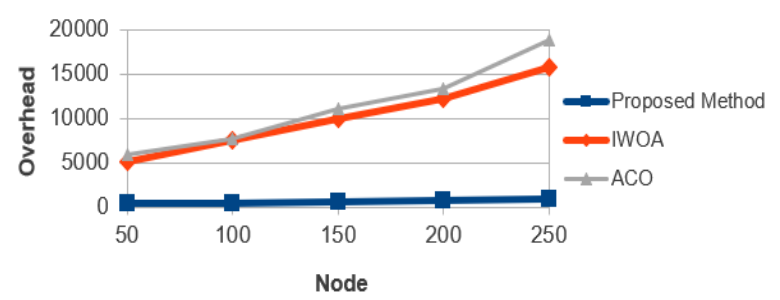

(a)

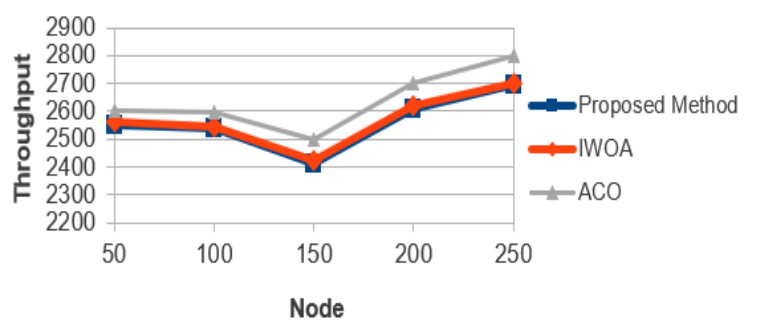

(b)

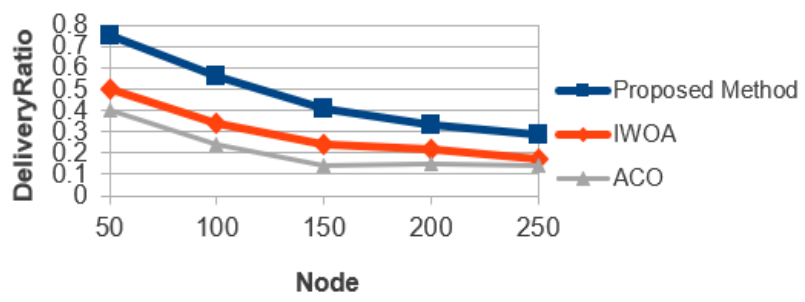

(c)

Fig.5: Comparison analysis of (a) Overhead (b) Throughput and (c) delivery ratio

Here, the VANETs are tested with the rectangular and circular path; in this scenario, 250/500 vehicles are utilized to analyze the routing overhead, throughput and delivery ratio of the VANETs and Figure 6 shows overhead, throughput and delivery ratio concerning time frame. In figure 6(a) as time increases the overhead increases slightly, but our method shows significantly less overhead as compared to other methods. It determines overhead by the analysis of transferring 
messages. In figure 6(b), our approach shows better throughput as compared to other existing methods. In figure $6(\mathrm{c})$ the delivery ratio is excellent and steady as compared to Ant Colony Optimization (ACO) and Improved Whale Optimization algorithm (IWOA).

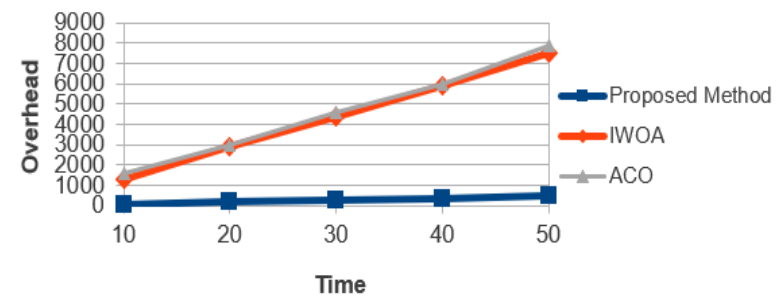

(a)

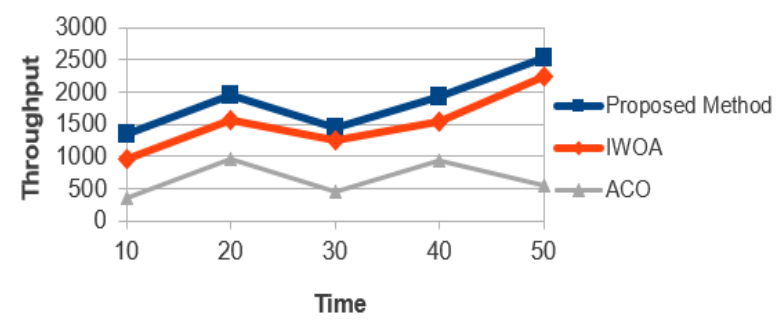

(b)

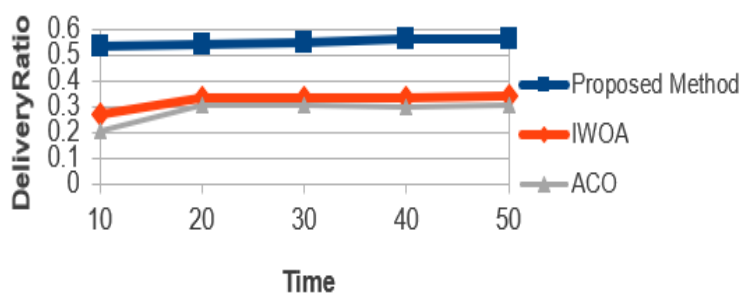

(c)

Fig.6: Comparison analysis of (a) Overhead (b) Throughput and (c) delivery ratio

\section{Conclusion}

In this paper, we have developed Clustering Adaptive Elephant Herd Optimization Based Data Dissemination Protocol for VANETs. The protocol uses the concept of clustering and routing procedures simultaneously. It helps us to find the shortest and stable routes between the source and the destination. Optimize route selection is based on parameters which are evaluated by the fitness function. These parameters make changes in the optimal outcomes in the AODV routing protocol and it also supports the efficient data dissemination between clusters. Our proposed algorithm is compared with existing algorithms such as ACO and IWOA on NS2 platform; the results show that our algorithm generates better results in term of throughput and Packet Delivery ratio with reduced routing overhead.

\section{References}

1) Ammara Anjum Khan, Mehran Abolhasan and Wei $\mathrm{Ni}$, “An Evolutionary Game Theoretic Approach for Stable and Optimized Clustering in VANETs", IEEE Transactions on Vehicular Technology,67(5),4501-4513,doi:10.1109 tvt.2018.2790391.

2) Andrea Baiocchi; Pierpaolo Salvo; Francesca Cuomo; Izhak Rubin, "Understanding Spurious Message Forwarding in VANET Beaconless Dissemination Protocols: An Analytical Approach”, IEEE Transactions on Vehicular Technology, Vol. 65, (No. 4),(April 2016).

3) Ata Ullah, ShumaylaYaqoob "Emergency Message Dissemination Schemes Based on Congestion Avoidance in VANET and Vehicular FoG Computing”, Advanced Big Data Analysis For Vehicular Social Networks, (2019).

4) Rejab Hajlaoui, Hervé Guyennet, Tarek Moulahi “A survey on Heuristic-based Routing Methods in Vehicular Ad-Hoc Network: Technical challenges and future trends”, 1530-437X (c) 2016 IEEE, DOI10.1109/JSEN.2016.2583382, IEEE Sensors Journal.

5) Celimuge WU, Satoshi Ohzahata and Toshihiko Kato, "VANET Broadcast protocol based on fuzzy logic and lightweight retransmission mechanism", IEICE Trans.Commun., Vol. E95-B, (No. 2), (2012).

6) Shilpa Choudhary, Abhishek Sharma, Kashish Srivastava, Hemant Purohit, Mudita Vats, " Read Range Optimization of Low Frequency RFID System in Hostile Environmental Conditions by Using RSM Approach,” EVERGREEN Joint Journal of Novel Carbon Resource Sciences \& Green Asia Strategy, 7(3) 396-403 (2020).

7) Alireza Askarzadeh "A novel metaheuristic method for solving constrained engineering optimization problems: Crow search algorithm”, http://dx.doi.org/10.1016/j.compstruc.2016.03.001, 0045-7949/@ 2016 Elsevier Ltd.

8) Fanhui Zeng; Rongqing Zhang; Xiang Cheng; Liuqing Yang, "Channel Prediction Based Scheduling for Data Dissemination in VANETs “, IEEE Communications Letters, Vol. 2, (No. 6, pp. 1409-1412), (June 2017).

9) Ryan Imansyah, " Impact of Internet Penetration for the Economic Growth of Indonesia,” EVERGREEN Joint Journal of Novel Carbon Resource Sciences \& Green Asia Strategy, 5(2) 36-43 (2018).

10) Jianping He, Lin Cai, Peng Cheng, and Jianping Pan, "Delay Minimization for Data Dissemination in Large-scale VANETs with Buses and Taxis”, IEEE, (2015).

11) Jianping He, Yuanzhi Ni, Lin Cai, Jianping Pan and Cailian Chen, “Optimal Dropbox Deployment 
Algorithm for Data Dissemination in Vehicular Networks", IEEE Transactions on Mobile Computing, Vol. 17, (No. 3), (2018).

12) Lei Liu; Tie Qiu;Chen Chen; Mengyuan Zhang, “A Data Dissemination Scheme based on Clustering and Probabilistic Broadcasting in VANETs”, Vehicular Communications 13, (pp:78-88) (May 2018).

13) Manisha Chahal and SandeepHarit, "Optimal path for data dissemination in Vehicular Ad Hoc Networks using meta-heuristic", Computers \& Electrical Engineering, Vol. 76, (pp. 40-55), (June 2019).

14) Mohammed Ali Berawi, Sutan Akbar Onggar Siahaan, Gunawan, Miraj, Perdana "Determining the Prioritized Victim of Earthquake Disaster Using Fuzzy Logic and Decision Tree Approach" Evergreen. 7 (2), pp.246-252, (2020-06).

15) Asad Ali, Syed Adeel Ali Shah, "VANET Clustering using whale optimization algorithm", 978-1-7281-3073-6/19/\$31.00 @2019.

16) Sabri Allani, Taoufik Yeferny, Richard Chbeir, Sadok Ben Yahia "A Novel VANET Data Dissemination Approach Based on Geospatial Data” @ 2016 Published by Elsevier B.V this is an open access article under the CC BY-NC-ND license.

17) Dief, Tarek N, Yoshida, Shigeo, "System Identification for Quad-rotor Parameters Using Neural Network", Evergreen. 3 (1), pp.6-11, (2016-03).

18) Rasmeet S.Bali and NeerajKumar, "Secure clustering for efficient data dissemination in vehicular cyber-physical systems", Future Generation Computer Systems, Vol. 56, (pp. 476-492), (March 2016).

19) Taofeek A. O. Yusuf, D. T. Altilar, "Impact on Inter-Vehicular Communication Performance on Different Traffic Mobility Model:A Case Study of Ad Hoc On-Demand Distance Vector Routing Protocol”(C2017ACM,97814503530901707,DOI: 10.1145/3129186.3129188.

20) R. Yarinezhad and A. Sarabi, “A New Routing Algorithm for Vehicular Ad-hoc Networks based on Glowworm Swarm Optimization Algorithm”, Journal of AI and Data Mining, Vol. 7, No 1, (pp. 69-76), (2019).

21) Jinhwan Jang "Outlier filtering algorithm for travel time estimation using dedicated short-range communications probes on rural highways" IET Intell. Transp. Syst., 2016, Vol. 10, Iss. 6, pp. 453460 \& the Institution of Engineering and Technology 2016.

22) Weina Zhang Ruijuan Zheng, Mingchuan Zhang, Junlong Zhu and Qingtao Wu, "ECRA: An Encounter-aware and Clustering-based Routing Algorithm for Information-centric VANETs", Mobile Networks and Applications, (pp. 1-11), (2019).
23) Lan Wang, Vince Lehman, A K M Mahmudul Hoque, Beichuan Zhang "A Secure Link State Routing Protocol for NDN” 2169-3536 (c) 2017 IEEE. DOI 10.1109/ACCESS.2017.2789330.

24) $P$. Tungjiratthitikan, "Accidents in thai industry between 2001 and 2017,” Evergreen, (2018). doi:10.5109/1936221.

25) Zhiyuan Li and Yue SongJunlei Bi, "CADD: connectivity-aware data dissemination using node forwarding capability estimation in partially connected VANETs", Wireless Networks, Vol. 25, No. 1, (pp. 379-398, January 2019). 